EXPERIMENTAL MODEL

\section{FASD: new insights from zebrafish}

Weeks, $\mathrm{O}$ et al. J. Clin. Invest. https://doi.

org/10.1172/JCI132139 (2020)

Fetal alcohol spectrum disorders (FASD) result from prenatal alcohol exposure and affect $1-5 \%$ of US school-age children. Patients with FASD present with distinctive features such as facial characteristics, microcephaly, cognitive deficits, and organ malformations, but the metabolic effects of the condition are not well understood. A study used zebrafish to model FASD and assess the metabolic consequences of embryonic alcohol exposure (EAE) in fish fed a high-fat, high-cholesterol diet. EAE zebrafish had more features of metabolic syndrome (MS) such as visceral adiposity, elevated BMI and fasting hyperglycemia than control zebrafish which were not exposed to ethanol. The findings also suggest that reduced locomotor activity, abnormal hepatic development, and transcriptional changes drive MS development in FASD.

\section{IMMUNOLOGY}

\section{Atlas of immune cells in Drosophila}

Cattenoz, P.B. et al. EMBO J. e104486 (2020)

Three types of hemocytes - the mediators of innate immunity in invertebrateshave been identified in Drosophila: plasmatocytes, which are responsible for the main functions of the hemocytes, crystal cells accounting for $<5 \%$ of hemocytes, and lamellocytes, which only appear upon challenge.

A new study published in $E M B O$ Journal provides further insights into the temporal specificity and heterogeneity of these cells. Bulk RNA sequencing of hemocytes from embryos and larvae revealed the transcriptional changes occurring during development, and singlecell RNA sequencing identified 14 hemocyte clusters present in larvae. The atlas, which also describes the transcriptional changes occurring in the hemocyte clusters upon wasp infestation, is an important resource to understand the role of these immune cells in physiological and pathological conditions.

\section{AGING}

\section{Transcriptomic effects of diet}

Ma, S. et al. Cell 180, 984-1001 (2020)

Aging elicits different responses across cell types, tissues and organs, which hampers our understanding of this process. Caloric restriction (CR) consistently increases lifespan across multiple species, but the mechanisms through which CR exerts this effect are also not well understood. In a new study published in Cell, investigators compared the transcriptomic profile of 210,000 single cells and nuclei across nine types of tissues from young and old rats (Rattus Norvegicus) subjected to ad libitum feeding, and old rats subjected to CR. The transcriptomic atlas uncovered ageassociated features at the molecular, cellular, tissue, and organismal levels, which were rescued by CR. These findings enhance our understanding of aging, confirm the powerful effects $C R$, and could inform new geroprotective interventions.

https://doi.org/10.1038/s41684-020-0544-y

\section{STROKE \\ VEGFD delivery for stroke therapy}

Mauceri, D. et al. PNAS https://doi.org/10.1073/

pnas.2001563117 (2020)

Thrombolytic therapy, the only available treatment for stroke, is not suitable for all patients; new therapies are therefore needed to improve motor and cognitive functions in stroke survivors.

Hypothesizing that a therapeutic strategy aimed at preserving dendritic connections could improve stroke outcomes, a group of investigators from Heidelberg University assessed the effect of VEGFD delivery in the mouse model of middle cerebral artery occlusion. Previous studies had shown that VEGFD, a growth factor expressed in the brain, is involved in dendrite maintenance.

Supplementation of VEGFD mitigated the stroke-induced loss of dendritic structure, thereby preserving the integrity of the neurons and their synaptic connectivity. Administration of VEGFD also reduced brain damage and improved functional recovery after experimental stroke, demonstrating the therapeutic potential of this factor.

https:/doi.org/10.1038/s41684-020-0546-9

Alexandra Le Bras and Ellen P. Neff nature masterclasses

\section{Online Course in Scientific Writing and Publishing}

Delivered by Nature Research journal editors, researchers gain an unparalleled insight into how to publish.

\section{Try a free sample of the course at masterclasses.nature.com}

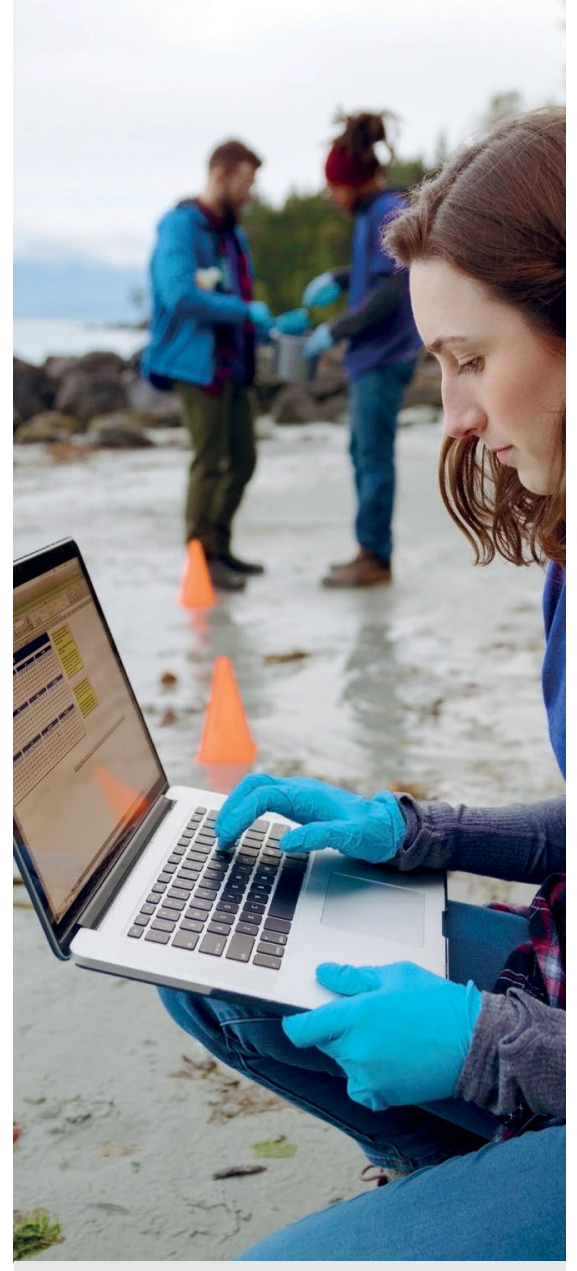

Bite-size design for busy researchers Subscribe as a lab or institution

W masterclasses.nature.com

(in Follow us on Linkedln

f Skills and Careers Forum for Researchers 